\title{
Correction to: Osteopontin Signaling in Shaping Tumor Microenvironment Conducive to Malignant Progression
}

Ramesh Butti, Totakura V. S. Kumar, Ramakrishna Nimma, Pinaki Banerjee, Ipsita G. Kundu, and Gopal C. Kundu

Correction to:

Chapter 20 in: A. Birbrair (ed.), Tumor Microenvironment, Advances in Experimental Medicine and Biology 1329, https://doi.org/10.1007/978-3-030-73119-9_20

Fig. 3 was misrepresented as both Fig. 3 and Fig. 4 in duplicated ways. The original

Fig. 3 was missing in the final version which has now been updated. 


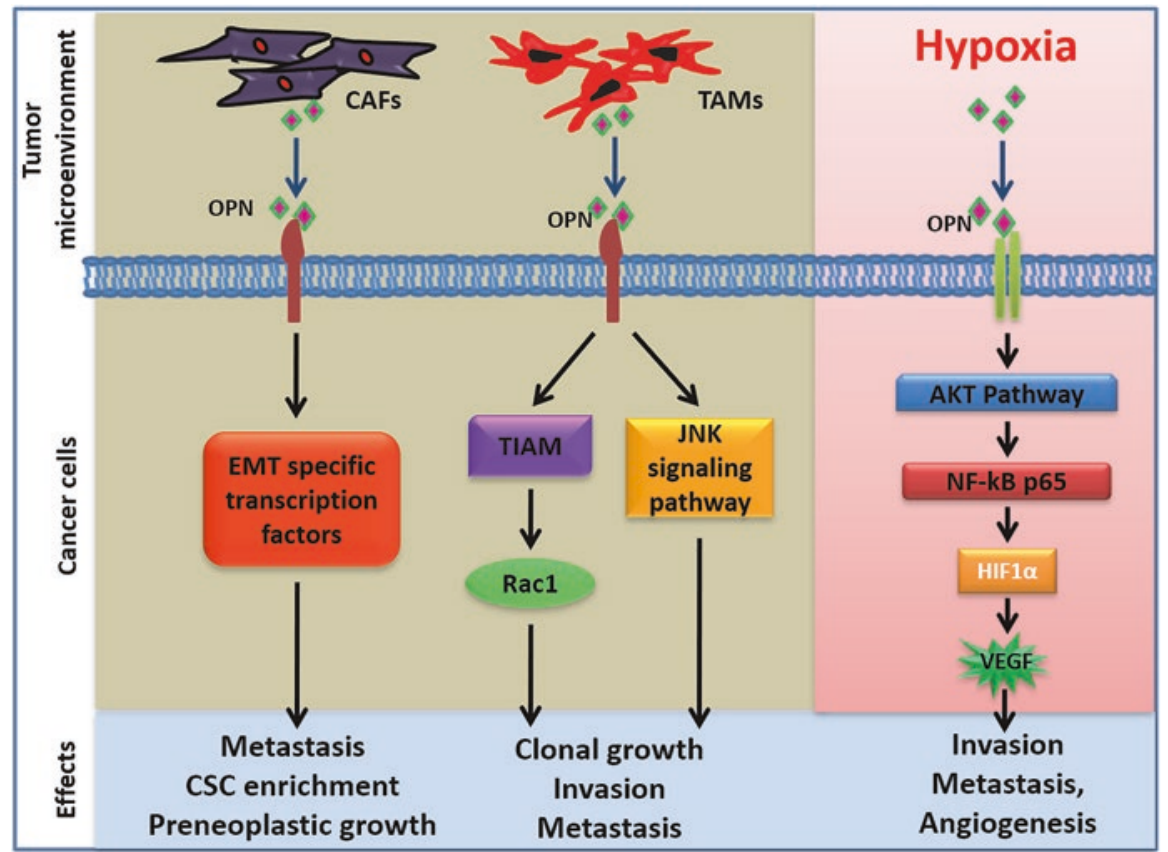

Fig. 3 Stromal cell- and hypoxia-derived OPN-induced signaling in cancer cells. OPN secreted from CAFs promotes invasion, metastasis, and CSC phenotype by inducing the expression of EMT-specific transcription factors. TAMs promote cancer cell invasion, metastasis, and clonal growth by secreting OPN. OPN secreted by TAMs induce both TIAM1 and JNK signaling pathway in cancer cells. Hypoxia induces the expression of OPN that leads to the upregulation of VEGF through HIF1 $\alpha$ by activation of AKT pathway. This in turn leads to the induction of tumor angiogenesis and metastasis 